\title{
Planned Activity Study Day Range
}

National Cancer Institute

\section{Source}

National Cancer Institute. Planned Activity Study Day Range. NCI Thesaurus. Code C95379.

The intended study day range of the activity. 